\section{The BCS Annual Conference, Manchester, 8-10 June 2015. The vice-president's message}

\author{
Clifford Garratt
}

It is an exciting time for the British Cardiovascular Society (BCS) team as the opening of the 2015 conference approaches. I have attended this meeting for many years both in its former heyday and, more recently, in its resurgence as the most comprehensive high-quality cardiovascular meeting in the UK. The meetings of the last few years have set an increasingly high standard, and this trajectory is set to continue for 2015 and beyond.

\section{THEME OF 'HEART AND GENES'}

This year, our theme is 'Hearts and Genes', and several of the sessions share this focus. The conference will be opened by the president, Dr Iain Simpson, followed by the opening lecture on 'where are we going with molecular medicine' delivered by Professor Robert Winston (figure 1).

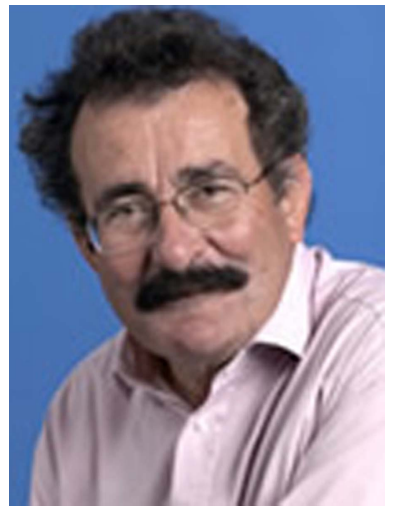

Figure 1 Professor Robert Winston

\section{NEW SCIENTIFIC SESSIONS IN COLLABORATION WITH THE BHF}

The British Heart Foundation is a key supporter of the annual conference, and this collaboration has increased significantly for 2015, with new session formats being introduced. Professor Hugh Watkins (figure 2) will facilitate the first of these sessions focusing on hypertrophic

Correspondence to Professor Clifford Garratt, Manchester Heart Centre, Manchester Royal Infirmary, Central Manchester University Foundation Trust, Manchester M13 9WL, UK; clifford.garratt@cmft.nhs.uk

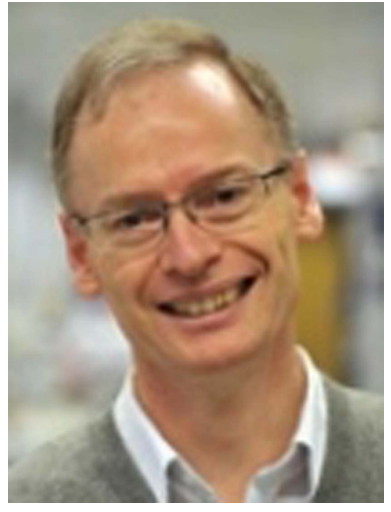

Figure 2 Professor Hugh Watkins

cardiomyopathy. The session will consist of four talks on different aspects of the condition; all presented by members of the same unit, encompassing basic, translational and clinical aspects of the disease. The purpose of the session is to provide a coordinated, in-depth review of current knowledge of the condition and to give an insight into the vision and organisation of a successful clinical research department. We think that this aspect will be of particular interest to trainees considering research at some stage in their careers, and for this reason, the Training Day programme will combine with this session in the auditorium on the first morning of the conference.

The BHF and the UK Genetic Testing Network are supporting two other sessions on the first day, both related to the new genetic testing panels for cardiovascular disease. Experts in cardiovascular genetics from a number of different fields (including cardiomyopathies, sudden death syndromes, familial hypercholesterolaemia and pulmonary hypertension) will give an insight into the clinical utility of these tests, and the role of whole genome sequencing will also be addressed (Professor Bernard Keavney).

\section{INTERACTIVE CLINICAL SESSIONS ('MDT LIVE')}

These sessions focus on the practical issues of diagnosis and management of cardiovascular conditions and are in the form of live multidisciplinary team discussion of clinical cases. 'Blind cases' will be presented with clinical and imaging data to a panel of both subspecialist and 'generalist' experts, including surgeons, imaging cardiologists and allied professionals. Interaction with the audience is encouraged, and each session will have a single facilitator who is tasked with ensuring that the important clinical messages are brought to light. This format has been successful at previous conferences, but is significantly expanded this year, with sessions in the auditorium on each of the 3 days, covering intervention (Dr Mike Norell), arrhythmias (Dr Derick Todd) and heart failure (Professor Andrew Clark).

\section{OTHER NEW FORMATS FOR 2015}

The standard format of medical conference sessions, with a series of talks followed by audience questions, has its place, but we recognise that shaking this format up a bit brings rewards in terms of entertainment, enjoyment and getting across appropriate messages. Tuesday afternoon has a number of new-format sessions, including one chaired by Professor Roger Hall in which cardiologists will be quizzed on illustrative medicolegal cases by Sally Smith QC (figure 3). Professor Peter Sleight will be discussing the effects of musical rhythms on the cardiovascular system in a session featuring both illustrative clips of classical music and live performances from the Welsh Cardiovascular Society male voice choir. In 'The Transplant Cycle', Professor Greg Whyte and Dr John Buckley will return to the Manchester stage, telling the true story of a cardiac transplant recipient who is making the most of his new heart.

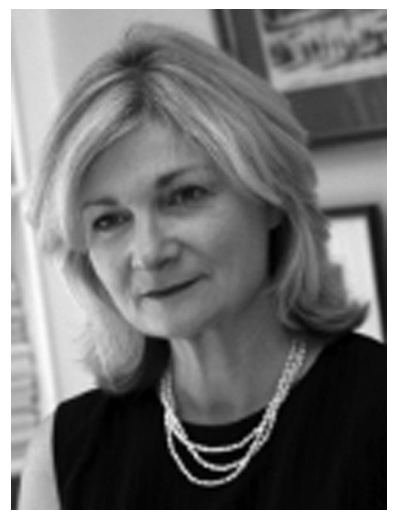

Figure 3 Sally Smith QC

THE SPRING MEETING OF THE BRITISH SOCIETY OF CARDIOVASCULAR RESEARCH AND BRITISH ATHEROSCLEROSIS SOCIETY

The 'basic science track' is provided by the British Society of Cardiovascular 
Research/British Atherosclerosis Society's (BSCR/BAS) Spring meeting, integrated into the first 2 days of the conference. This part of the meeting is always extremely well attended and of very high quality in terms of scientific content. This year's focus is on new frontiers in cardiovascular science with basic science sessions, including (1) altering blood vessel growth to reduce cardiovascular disease, (2) vascular calcification, (3) systemic inflammation and cardiovascular disease and (4) unravelling the structural basis of cardiovascular disease through the application of advanced imaging techniques.

\section{Other highlights of the meeting}

- Named lectures from Professor Jonathan Kalman (figure 4) and Sir Harry Burns.

- National training day, including Specialist Advisory Committee (SAC) session.

- Imaging: dedicated imaging track, abstract poster sessions and integrated imaging content in the highlight sessions of the programme.

- Arrhythmias: sessions on AF, inherited arrhythmias, guidelines on prevention of device infection as well as arrhythmia abstract sessions, discussion of arrhythmia clinical cases, the Paul Wood lecture from Professor Jonathan Kalman and a whole track of arrhythmia hot topics throughout the 3 days.

- Intervention: sessions on acute coronary syndromes, myocardial infarction, valve disease intervention as well as Acute Coronary Syndromes (ACS) and intervention abstract sessions, discussion of interventional clinical cases and a whole track of interventional hot topics throughout the 3 days.

- Heart failure: sessions on acute heart failure, cardiomyopathies, the heart and systemic disease, cardio-oncology, guidelines on prevention of device infection as well as heart failure abstract sessions, discussion of heart failure clinical cases and a whole track of heart failure hot topics throughout the 3 days.

- Adult Congenital Heart Disease (ACHD) sessions on European Society of Cardiology (ESC) guidelines on hypertrophic cardiomyopathy (HCM) and aortic disease, a British Congenital Cardiologists Association (BCCA) session,

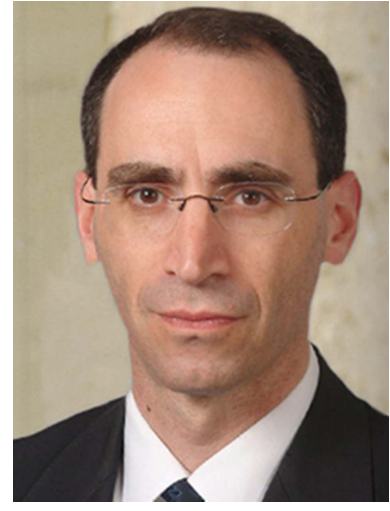

Figure 4 Professor Jonathan Kalman

shared care in ACHD together with ACHD abstracts and hot topics.

- Clinical science translational research (CSTR), including the Young Research Workers Prize (YRWP), the Michael Davies Early Career Award and abstract poster sessions.

- Education for revalidation (E4R) sessions, including 'Top 10 Trials' and 'Life Long Learning'.

- Multisession tracks dedicated to British Heart Foundation (BHF) healthcare professionals, British Association for Cardiovascular Prevention and Rehabilitation (BACPR)/British Association for Nursing in Cardiac Care (BANCC)/British Society for Heart Failure (BSH), members, patients (Cardiac Care Partnership United Kingdom (CCPUK)) and healthcare scientists.

- Session on 'Hypertension for cardiologists' provided by the British Hypertension Society with a line-up of internationalquality speakers.

The 2015 annual conference will feature a dedicated area for interactive education provided by the BCS, the BHF and industry colleagues; this year's education hall will feature:

- Poster area: interactive poster/abstract sessions.

- Hot topics: back by popular demand, these short, interactive presentations cover the latest FAQs in cardiology.

- Simulation: The year 2015 will see a variety of technical simulators available, as well as the essential surgical skills station, with supervised training by experienced consultants.
- Imaging village: interactive, supervised work stations for CT, nuclear cardiology, MRI and echo will be available with opportunities to undertake scans on volunteers.

- Resuscitation sessions: interactive sessions facilitated by an expert faculty and The Resuscitation Council (UK).

- Annual dinner: now a highlight of the conference, this year's dinner will be bigger than ever, with a champagne reception, three-course dinner and a fundraising raffle. The BHF Alliance awards will be presented to the winners at the annual dinner. The awards, which celebrate high-quality services and care, are awarded to members of the BHF Alliance who champion the BHF's values and mission to win the fight against cardiovascular disease. Tickets are available for purchase when registering for the conference.

Finally, I have been hugely impressed by the willingness of cardiologists and cardiovascular scientists, both from the UK and internationally, to give time and effort to the planning of this meeting. In particular, I would like to acknowledge the help of the BHF team and the staff, officers and Programme Committee of the BCS. Dr Sarah Clarke, outgoing programme chair, and Steven Yeats (BCS chief executive) have been particularly helpful in terms of easing me gently into the programme-chair role. The full programme and booking for lifelong learning, simulation, imaging village, resuscitation skills sessions and the annual dinner are available online at http://www. bcs.com/annualconference. An app containing the programme and biographies of the faculty is also available (BCS Conf. 2015). I have absolutely no doubt that this year's conference will be the most enjoyable and educational yet. The only remaining question is, how will we match it in 2016 ?

\section{Competing interests None declared.}

Provenance and peer review Commissioned; internally peer reviewed.

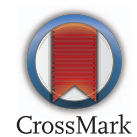

To cite Garratt C. Heart 2015;101:826-827.

Heart 2015;101:826-827.

doi:10.1136/heartjnl-2015-307932 